\title{
Ekeland's variational principle in Fréchet spaces and the density of extremal points
}

\author{
by
}

\author{
J. H. QIU (Suzhou)
}

\begin{abstract}
By modifying the method of Phelps, we obtain a new version of Ekeland's variational principle in the framework of Fréchet spaces, which admits a very general form of perturbations. Moreover we give a density result concerning extremal points of lower semicontinuous functions on Fréchet spaces. Even in the framework of Banach spaces, our result is a proper improvement of the related known result. From this, we derive a new version of Caristi's fixed point theorem and a density result for Caristi fixed points.
\end{abstract}

1. Introduction. Ekeland proved $[4,5]$ an important theorem on complete metric spaces, called the variational principle, which has many applications in nonlinear analysis. By using an $X \times \mathbb{R}$ version of a classical maximality result due to Bishop and Phelps, Phelps [11, p. 47] obtained the following useful version of Ekeland's variational principle in Banach spaces.

Theorem 1.1 (see [11, Lemma 3.13]). Let (X, \|\|) be a Banach space and $f:(X,\|\|) \rightarrow \mathbb{R} \cup\{\infty\}$ be a lower semicontinuous proper function, bounded from below. Suppose that $\varepsilon>0$ and that $f\left(x_{0}\right)<\inf f(X)+\varepsilon$. Then for any $\lambda$ with $0<\lambda<1$, there exists a point $z \in \operatorname{dom}(f)$ such that

(i) $\lambda\left\|z-x_{0}\right\| \leq f\left(x_{0}\right)-f(z)$,

(ii) $\left\|z-x_{0}\right\|<\varepsilon / \lambda$,

(iii) $\lambda\|x-z\|+f(x)>f(z)$, whenever $x \neq z$.

Here $\inf f(X)$ denotes $\inf \{f(x): x \in X\}$, and a function $f: X \rightarrow$ $\mathbb{R} \cup\{\infty,-\infty\}$ is said to be proper if its effective domain, i.e. $\operatorname{dom}(f)=$ $\{x \in X: f(x) \neq \infty$ and $f(x) \neq-\infty\}$, is nonempty. Given a function $f: X \rightarrow \mathbb{R}$ and a positive real number $\lambda$, as in [1] we put

$$
E_{f, \lambda}=\{z \in X: f(x)+\lambda\|x-z\|>f(z), \forall x \in X, x \neq z\} .
$$

A point in $E_{f, \lambda}$ is called a $\lambda$-extremal point of $f$. Cammaroto and Chinni [1] considered the density of extremal points and established the following qualitative complement to Ekeland's variational principle.

2000 Mathematics Subject Classification: 49J45, 46A04.

Key words and phrases: Ekeland's variational principle, Fréchet space, extremal points, perturbations. 
TheOREM 1.2 (see [1, Theorem 1]). Let $(X,\|\|)$ be a Banach space and $f:(X,\|\|) \rightarrow \mathbb{R}$ a lower semicontinuous function, bounded from below. Assume that

$$
\limsup _{\|x\| \rightarrow \infty} \frac{f(x)}{\|x\|}<\infty .
$$

Then for every $\lambda>\lim \sup _{\|x\| \rightarrow \infty} f(x) /\|x\|$, one has $\overline{\operatorname{conv}}\left(E_{f, \lambda}\right)=X$, where $\overline{\operatorname{conv}}\left(E_{f, \lambda}\right)$ denotes the closed convex hull of $E_{f, \lambda}$.

In this paper, by modifying the proof of Phelps we obtain a very general version of Ekeland's variational principle (see Theorem 2.1), which not only extends Theorem 1.1 from Banach spaces to Fréchet spaces (i.e. complete countable seminormed locally convex spaces), but also has a more general form of perturbations. Moreover, we give a density result concerning extremal points for lower semicontinuous functions defined on Fréchet spaces, which is a generalization of Theorem 1.2 (see Theorem 3.1). In fact, the condition we need to assume is strictly weaker than the condition in Theorem 1.2 even in the framework of Banach spaces. Hence the density result we obtain is a proper improvement of Theorem 1.2. Finally, from the above-mentioned results we derive a new version of Caristi's fixed point theorem (see [2]) and a density result for Caristi fixed points.

2. A general version of Ekeland's variational principle. Let $\varphi$ : $[0, \infty) \rightarrow[0, \infty)$ be a subadditive, strictly increasing, continuous function with $\varphi(0)=0$ and let the class $\Theta$ consist of all such functions $\varphi$. Clearly, the converse of $\varphi, \varphi^{-1}: \varphi([0, \infty)) \rightarrow[0, \infty)$, exists and is a superadditive, strictly increasing, continuous function with $\varphi^{-1}(0)=0$. Here $\varphi$ is said to be subadditive if

$$
\varphi(t+s) \leq \varphi(t)+\varphi(s), \quad \forall t, s \in[0, \infty),
$$

and $\varphi^{-1}$ is said to be superadditive if

$$
\varphi^{-1}(x+y) \geq \varphi^{-1}(x)+\varphi^{-1}(y), \quad \forall x, y \in \varphi([0, \infty)) .
$$

We can verify that the following functions belong to $\Theta$ :

$$
\begin{aligned}
\varphi(t) & =\lambda t, & & \text { where } \lambda>0 \text { is a constant; } \\
\varphi(t) & =\frac{\alpha t}{1+\beta t}, & & \text { where } \alpha, \beta>0 \text { are constants; } \\
\varphi(t) & =\alpha \ln (1+\beta t), & & \text { where } \alpha, \beta>0 \text { are constants; } \\
\varphi(t) & =\alpha \sqrt[n]{t}, & & \text { where } \alpha>0 \text { and } n \in \mathbb{N} \text { are constants; } \\
\varphi(t) & =\alpha \arctan (\beta t), & & \text { where } \alpha, \beta>0 \text { are constants; } \\
\varphi(t) & =\sin \left(\frac{\pi}{4} \cdot \frac{\alpha t}{1+\beta t}\right), & & \text { where } \alpha \text { and } \beta \text { are constants, } 0<\alpha \leq \beta .
\end{aligned}
$$


Let $(X, \mathcal{T})$ be a locally convex Hausdorff topological vector space (briefly, a locally convex space) and $p$ a continuous seminorm on $(X, \mathcal{T})$. For $\varphi \in \Theta$, we define

$$
K_{\varphi, p}=\{(x, r) \in X \times \mathbb{R}: \varphi(p(x)) \leq-r\} .
$$

Obviously $(0,0) \in K_{\varphi, p}$ and $K_{\varphi, p}$ is closed in the product topology of $X \times \mathbb{R}$. Clearly

$$
K_{\varphi, p}+K_{\varphi, p} \supset K_{\varphi, p}
$$

since $(0,0) \in K_{\varphi, p}$. On the other hand, if $(x, r),\left(x^{\prime}, r^{\prime}\right) \in K_{\varphi, p}$, then

$$
\varphi\left(p\left(x+x^{\prime}\right)\right) \leq \varphi\left(p(x)+p\left(x^{\prime}\right)\right) \leq \varphi(p(x))+\varphi\left(p\left(x^{\prime}\right)\right) \leq-\left(r+r^{\prime}\right),
$$

which implies that $(x, r)+\left(x^{\prime}, r^{\prime}\right)=\left(x+x^{\prime}, r+r^{\prime}\right) \in K_{\varphi, p}$. Thus

$$
K_{\varphi, p}+K_{\varphi, p} \subset K_{\varphi, p}
$$

and so $K_{\varphi, p}+K_{\varphi, p}=K_{\varphi, p}$.

Lemma 2.1. Let $(X, \mathcal{T})$ be a Fréchet space with topology generated by an increasing sequence \|\|$_{1} \leq\|\|_{2} \leq \cdots$ of seminorms on $X$ (for example, see [7]). For a sequence $\left(\varphi_{n}\right)_{n \in \mathbb{N}} \subset \Theta$, define a decreasing sequence $K_{1} \supset$ $K_{2} \supset \cdots$ of closed sets in $X \times \mathbb{R}$ as follows:

$$
K_{n}=\left\{(x, r) \in X \times \mathbb{R}: \varphi_{i}\left(\|x\|_{i}\right) \leq-r, 1 \leq i \leq n\right\} .
$$

Assume that $A \subset X \times \mathbb{R}$ is a nonempty closed set and $\inf \{r:(x, r) \in A\}=0$. Then for any $\left(x_{0}, r_{0}\right) \in A$ and $i \in \mathbb{N}$, there exists $(z, r) \in A \cap\left(K_{i}+\left(x_{0}, r_{0}\right)\right)$ such that

$$
\{(z, r)\}=A \cap \bigcap_{n=1}^{\infty}\left(K_{n}+(z, r)\right) .
$$

Proof. We let $g: X \times \mathbb{R} \rightarrow \mathbb{R}$ be the projection. For any nonempty subset $B$ of $X \times \mathbb{R}$, we denote $\inf \{r:(x, r) \in B\}$ by $\inf g(B)$. Without loss of generality, we may assume that $i=1$. Put $A_{1}:=A \cap\left(K_{1}+\left(x_{0}, r_{0}\right)\right)$. Then $A_{1} \subset A$ and

$$
\inf g\left(A_{1}\right) \geq \inf g(A)=\inf \{r:(x, r) \in A\}=0 .
$$

Choose $\left(x_{1}, r_{1}\right) \in A_{1}$ such that

$$
r_{1}<\inf g\left(A_{1}\right)+1 / 2 .
$$

Put $A_{2}:=A \cap\left(K_{2}+\left(x_{1}, r_{1}\right)\right)$. Then $A_{2} \subset A$ and $\inf g\left(A_{2}\right) \geq \inf g(A)=0$. Choose $\left(x_{2}, r_{2}\right) \in A_{2}$ such that

$$
r_{2}<\inf g\left(A_{2}\right)+1 / 2^{2} .
$$

In general, put $A_{n}:=A \cap\left(K_{n}+\left(x_{n-1}, r_{n-1}\right)\right)$ and choose $\left(x_{n}, r_{n}\right) \in A_{n}$ such that

$$
r_{n}<\inf g\left(A_{n}\right)+1 / 2^{n} \text {. }
$$


We thus obtain a sequence $\left(x_{n}, r_{n}\right)_{n \in \mathbb{N}}$ in $X \times \mathbb{R}$ and a sequence $\left(A_{n}\right)_{n \in \mathbb{N}}$ of subsets of $X \times \mathbb{R}$ such that

$$
\left(x_{n}, r_{n}\right) \in A_{n}=A \cap\left(K_{n}+\left(x_{n-1}, r_{n-1}\right)\right)
$$

and

$$
r_{n}<\inf g\left(A_{n}\right)+1 / 2^{n} \quad \text { for every } n \in \mathbb{N} \text {. }
$$

It is clear that

$$
\begin{aligned}
A_{n+1} & =A \cap\left(K_{n+1}+\left(x_{n}, r_{n}\right)\right) \subset A \cap\left(K_{n+1}+K_{n}+\left(x_{n-1}, r_{n-1}\right)\right) \\
& \subset A \cap\left(K_{n}+K_{n}+\left(x_{n-1}, r_{n-1}\right)\right)=A \cap\left(K_{n}+\left(x_{n-1}, r_{n-1}\right)\right)=A_{n} .
\end{aligned}
$$

When $m \geq n+1$, we have

$$
\left(x_{m}, r_{m}\right) \in A_{m} \subset A_{n+1}=A \cap\left(K_{n+1}+\left(x_{n}, r_{n}\right)\right) .
$$

Thus $\left(x_{m}-x_{n}, r_{m}-r_{n}\right) \in K_{n+1}$, which implies that for $i=1, \ldots, n+1$,

$$
\begin{aligned}
0 & \leq \varphi_{i}\left(\left\|x_{m}-x_{n}\right\|_{i}\right) \leq r_{n}-r_{m}<\inf g\left(A_{n}\right)+1 / 2^{n}-\inf g\left(A_{m}\right) \\
& \leq \inf g\left(A_{n}\right)+1 / 2^{n}-\inf g\left(A_{n}\right)=1 / 2^{n} .
\end{aligned}
$$

Let $n_{0} \in \mathbb{N}$ and $\varepsilon>0$ be given. Since $\lim _{t \rightarrow 0^{+}} \varphi_{n_{0}}^{-1}(t)=0$, there exists $n_{\varepsilon} \geq n_{0}$ such that

$$
\varphi_{n_{0}}^{-1}\left(1 / 2^{n}\right)<\varepsilon \quad \text { for all } n \geq n_{\varepsilon} .
$$

When $m \geq n+1>n \geq n_{\varepsilon}$, we have

$$
0 \leq \varphi_{n_{0}}\left(\left\|x_{m}-x_{n}\right\|_{n_{0}}\right) \leq r_{n}-r_{m}<1 / 2^{n} \text {. }
$$

From this,

$$
\left\|x_{m}-x_{n}\right\|_{n_{0}}<\varphi_{n_{0}}^{-1}\left(1 / 2^{n}\right)<\varepsilon .
$$

Also, $0 \leq r_{n}-r_{m}<1 / 2^{n}$. Thus we conclude that $\left(x_{n}\right)_{n \in \mathbb{N}}$ and $\left(r_{n}\right)_{n \in \mathbb{N}}$ are Cauchy sequences in $(X, \mathcal{T})$ and in $\mathbb{R}$ respectively. Since $(X, \mathcal{T})$ and $\mathbb{R}$ are complete, there exist $z \in X$ and $r \in \mathbb{R}$ such that $x_{n} \rightarrow z$ and $r_{n} \rightarrow r(n \rightarrow \infty)$. For each $n \in \mathbb{N}, A_{n}=A \cap\left(K_{n}+\left(x_{n-1}, r_{n-1}\right)\right)$ is closed. Observe that $\left(x_{m}, r_{m}\right) \in A_{m} \subset A_{n}$ for every $m \geq n$ and $\left(x_{m}, r_{m}\right) \rightarrow(z, r)$ as $m \rightarrow \infty$. Hence $(z, r) \in A_{n}$ and

$$
(z, r) \in \bigcap_{n=1}^{\infty} A_{n}=A \cap \bigcap_{n=1}^{\infty}\left(K_{n}+\left(x_{n-1}, r_{n-1}\right)\right)
$$

In particular, $(z, r) \in A \cap\left(K_{1}+\left(x_{0}, r_{0}\right)\right)$. Next we show that

$$
\{(z, r)\}=A \cap \bigcap_{n=1}^{\infty}\left(K_{n}+(z, r)\right) .
$$

Since $(0,0) \in K_{n}$ for all $n \in \mathbb{N}$, we easily see that

$$
(z, r) \in A \cap \bigcap_{n=1}^{\infty}\left(K_{n}+(z, r)\right) \text {. }
$$


On the other hand, if

$$
\left(z^{\prime}, r^{\prime}\right) \in A \cap \bigcap_{n=1}^{\infty}\left(K_{n}+(z, r)\right),
$$

then

$$
\begin{aligned}
\left(z^{\prime}, r^{\prime}\right) & \in K_{n}+(z, r) \subset K_{n}+A_{n+1} \subset K_{n}+K_{n+1}+\left(x_{n}, r_{n}\right) \\
& \subset K_{n}+K_{n}+\left(x_{n}, r_{n}\right)=K_{n}+\left(x_{n}, r_{n}\right) .
\end{aligned}
$$

From this, $\left(z^{\prime}-x_{n}, r^{\prime}-r_{n}\right) \in K_{n}$, that is, for $i=1, \ldots, n$,

$$
\varphi_{i}\left(\left\|z^{\prime}-x_{n}\right\|_{i}\right) \leq r_{n}-r^{\prime} .
$$

Since $\left(z^{\prime}, r^{\prime}\right) \in K_{n}+(z, r) \subset K_{n}+K_{n}+\left(x_{n-1}, r_{n-1}\right)=K_{n}+\left(x_{n-1}, r_{n-1}\right)$ and $\left(z^{\prime}, r^{\prime}\right) \in A$, we have $\left(z^{\prime}, r^{\prime}\right) \in A \cap\left(K_{n}+\left(x_{n-1}, r_{n-1}\right)\right)=A_{n}$ and $r^{\prime} \geq \inf g\left(A_{n}\right)$. Combining this with (1), we have

$$
\begin{aligned}
0 \leq \varphi_{i}\left(\left\|z^{\prime}-x_{n}\right\|_{i}\right) & \leq r_{n}-r^{\prime}<\inf g\left(A_{n}\right)+1 / 2^{n}-\inf g\left(A_{n}\right) \\
& =1 / 2^{n}, \quad i=1, \ldots, n .
\end{aligned}
$$

From this, we see that $x_{n} \rightarrow z^{\prime}$ and $r_{n} \rightarrow r^{\prime}$. By the uniqueness of limits we conclude that $z^{\prime}=z$ and $r^{\prime}=r$. Thus the proof is complete.

By using Lemma 2.1, we can obtain an extension of Theorem 1.1.

TheOrem 2.1. Let $(X, \mathcal{T})$ be a Fréchet space with topology generated by an increasing sequence \|\|$_{1} \leq\|\|_{2} \leq \cdots$ of seminorms, and let $f:(X, \mathcal{T}) \rightarrow$ $\mathbb{R} \cup\{\infty\}$ be a lower semicontinuous proper function, bounded from below. Assume that $\varepsilon>0$ and $x_{0} \in X$ are such that $f\left(x_{0}\right)<\inf f(X)+\varepsilon$ and let $\left(\varphi_{n}\right)_{n \in \mathbb{N}} \subset \Theta$. Then for any $i \in \mathbb{N}$ there exists $z \in \operatorname{dom}(f)$ such that

(i) $\varphi_{j}\left(\left\|z-x_{0}\right\|_{j}\right) \leq f\left(x_{0}\right)-f(z)$ for $j=1, \ldots, i$;

(ii) $\varphi_{j}\left(\left\|z-x_{0}\right\|_{j}\right)<\varepsilon$ for $j=1, \ldots, i$;

(iii) for any $x \in X, x \neq z$, there exists $m \in \mathbb{N}$ such that

$$
\varphi_{m}\left(\|x-z\|_{m}\right)+f(x)>f(z),
$$

or equivalently, for any $x \in X, x \neq z$,

$$
\sup _{n} \varphi_{n}\left(\|x-z\|_{n}\right)+f(x)>f(z) .
$$

Proof. Since $f:(X, \mathcal{T}) \rightarrow \mathbb{R} \cup\{\infty\}$ is lower semicontinuous and bounded from below, we know that $A:=\operatorname{epi}(f)=\{(x, r): f(x) \leq r\}$ is closed in $X \times \mathbb{R}$ and $\inf f(X)>-\infty$. Without loss of generality, we may assume that $\inf f(X)=0$. Thus the assumption that $f\left(x_{0}\right)<\inf f(X)+\varepsilon$ becomes

$$
0 \leq f\left(x_{0}\right)<\varepsilon .
$$

Since $\left(x_{0}, f\left(x_{0}\right)\right) \in A$ and $\inf \{r:(x, r) \in A\}=0$, by Lemma 2.1, for any $i \in \mathbb{N}$ there exists

$$
(z, r) \in A \cap\left(K_{i}+\left(x_{0}, f\left(x_{0}\right)\right)\right.
$$


such that

$$
\{(z, r)\}=A \cap \bigcap_{n=1}^{\infty}\left(K_{n}+(z, r)\right) .
$$

Since $(z, r) \in A$ and $\inf f(X)=0$, we have

$$
0 \leq f(z) \leq r .
$$

By $(3),(z, r) \in K_{i}+\left(x_{0}, f\left(x_{0}\right)\right)$, hence $\left(z-x_{0}, r-f\left(x_{0}\right)\right) \in K_{i}$. From this, by applying (2) and (5), we see that for $j=1, \ldots, i$,

$$
\varphi_{j}\left\|x-z_{0}\right\|_{j} \leq f\left(x_{0}\right)-r \leq f\left(x_{0}\right)-f(z) \leq f\left(x_{0}\right)<\varepsilon .
$$

This means that $z \in \operatorname{dom}(f)$ satisfies (i) and (ii). Next we show that it satisfies (iii). First we assert that $r=f(z)$. It suffices to show that

$$
(z, f(z)) \in A \cap \bigcap_{n=1}^{\infty}\left(K_{n}+(z, r)\right)
$$

Clearly $(z, f(z)) \in A$. Hence if $(z, f(z)) \notin A \cap \bigcap_{n=1}^{\infty}\left(K_{n}+(z, r)\right)$, then there exists $n \in \mathbb{N}$ such that $(z, f(z)) \notin K_{n}+(z, r)$. This implies that $(0, f(z)-r) \notin K_{n}$, that is to say, there exists $i, 1 \leq i \leq n$, such that

$$
\varphi_{i}\left(\|0\|_{i}\right)>r-f(z),
$$

which leads to $f(z)>r$. This contradicts the fact that $(z, r) \in A$, i.e. $f(z) \leq r$. Thus

$$
(z, f(z)) \in A \cap \bigcap_{n=1}^{\infty}\left(K_{n}+(z, r)\right)=\{(z, r)\}
$$

and hence $r=f(z)$.

Now we can rewrite (4) as follows:

$$
\{(z, f(z))\}=A \cap \bigcap_{n=1}^{\infty}\left(K_{n}+(z, f(z))\right) .
$$

If $x \neq z$ and $f(x)=\infty$, then (iii) is certainly satisfied.

If $x \neq z$ and $f(x)<\infty$, then by $(7)$,

$$
(x, f(x)) \notin A \cap \bigcap_{n=1}^{\infty}\left(K_{n}+(z, f(z))\right) .
$$

Since $(x, f(x)) \in A$ we know that there exists $n \in \mathbb{N}$ such that $(x, f(x)) \notin$ $K_{n}+(z, f(z))$, which implies that

$$
(x-z, f(x)-f(z)) \notin K_{n} .
$$

Thus there exists $m, 1 \leq m \leq n$, such that

$$
\varphi_{m}\left(\|x-z\|_{m}\right)+f(x)>f(z) \text {. }
$$


In particular, if $\varphi_{n}(t)=\lambda_{n} t$, where $\lambda_{n}>0$ is a constant, then we have the following consequence.

COROLlary 2.1. Let $(X, \mathcal{T})$ be a Fréchet space with topology generated by an increasing sequence \|\|$_{1} \leq\|\|_{2} \leq \cdots$ of seminorms, and let $f:(X, \mathcal{T}) \rightarrow \mathbb{R} \cup\{\infty\}$ be a lower semicontinuous proper function, bounded from below. Assume that $\varepsilon>0$ and $x_{0} \in X$ are such that $f\left(x_{0}\right)<\inf f(X)+\varepsilon$ and let $\left(\lambda_{n}\right)_{n \in \mathbb{N}}$ be a sequence of positive real numbers. Then for any $i \in \mathbb{N}$, there exists $z \in \operatorname{dom}(f)$ such that

(i) $\lambda_{j}\left\|z-x_{0}\right\|_{j} \leq f\left(x_{0}\right)-f(z)$ for $j=1, \ldots, i$;

(ii) $\left\|z-x_{0}\right\|_{j}<\varepsilon / \lambda_{j}$ for $j=1, \ldots, i$;

(iii) for any $x \in X, x \neq z$, there exists $m \in \mathbb{N}$ such that

$$
\lambda_{m}\|x-z\|_{m}+f(x)>f(z),
$$

or equivalently, for any $x \in X, x \neq z$,

$$
\sup _{n} \lambda_{n}\|x-z\|_{n}+f(x)>f(z) .
$$

A generalization of the notion of subgradient, the notion of $\Phi$-subgradient, can also be applied to reformulate Ekeland's variational principle (see [9, Chapter 2]). Concerning $\Phi$-subgradients and their applications, see, for example, [3], [9] and [12]. Let $\Phi$ be the family of all upper semicontinuous, concave, proper functions on $X$ and let $\varphi: X \rightarrow \mathbb{R}$ be defined as follows:

$$
\varphi(x)=-\sup _{n} \lambda_{n}\|x-z\|_{n}, \quad \forall x \in X ;
$$

then $\varphi \in \Phi$. Thus Corollary 2.1(iii) can be rewritten as follows: for any $x \neq z, \varphi(x)-\varphi(z)<f(x)-f(z)$, that is to say, $\varphi(x)=-\sup _{n} \lambda_{n}\|x-z\|_{n}$ is a strict $\Phi$-subgradient of $f(x)$ at the point $z$. Similarly, from Theorem 2.1(iii), $\varphi(x)=-\sup _{n} \varphi_{n}\left(\|x-z\|_{n}\right)$ is a strict $\Psi$-subgradient of $f(x)$ at $z$. Here the function family $\Psi$ should be so large that $\varphi \in \Psi$.

3. Density results for extremal points. A point $z$ is called a $\left(\lambda_{n}\right)$ extremal point of $f$ if it satisfies (iii) of Corollary 2.1, that is, for any $x \neq z$, there is $m \in \mathbb{N}$ such that $\lambda_{m}\|z-x\|_{m}+f(x)>f(z)$. We set

$$
E_{f,\left(\lambda_{n}\right)}=\left\{z \in X: \forall x \in X, x \neq z, f(z)<f(x)+\sup _{n} \lambda_{n}\left\|z-x_{n}\right\|_{n}\right\} .
$$

Corollary 2.1 points out that if $f$ is a lower semicontinuous proper function, bounded from below, then for any sequence $\left(\lambda_{n}\right)_{n \in \mathbb{N}}$ of positive real numbers, $E_{f,\left(\lambda_{n}\right)} \neq \emptyset$. In this section we shall discuss the following problem: under what conditions is $E_{f,\left(\lambda_{n}\right)}$ dense in $X$ ? When $X$ is a Banach space, the problem was solved in [1]; see Theorem 1.2. We give a generalization of Theorem 1.2 to Fréchet spaces. We shall see that even in the framework of Banach spaces, our result (Theorem 3.1) is a proper improvement of Theorem 1.2. The following lemma is useful in our proof. 
Lemma 3.1 ([1, Proposition 1$])$. Let $X$ be a locally convex space, $X^{*}$ be its topological dual and $A$ be a nonempty subset of $X$. Then the following two statements are equivalent:

(a) $\overline{\operatorname{conv}}(A)=X$;

(b) for any $T \in X^{*} \backslash\{0\}$ and any $r \in \mathbb{R}$,

$$
A \cap(T \leq r) \neq \emptyset
$$

where $(T \leq r)$ denotes the set $\{x \in X: T(x) \leq r\}$.

TheOREM 3.1. Let $(X, \mathcal{T})$ be a Fréchet space with topology generated by an increasing sequence \|\|$_{1} \leq\|\|_{2} \leq \cdots$ of seminorms, and let $f:(X, \mathcal{T})$ $\rightarrow \mathbb{R}$ be a lower semicontinuous function, bounded from below, which satisfies

$$
M_{n}:=\sup _{x \in X} \sup _{\substack{y \in X \\\|y\|_{n}=1}} \liminf _{t \rightarrow \infty} \frac{f(x+t y)}{t}<\infty, \quad \forall n \in \mathbb{N} .
$$

Let $\left(\lambda_{n}\right)_{n \in \mathbb{N}}$ be a sequence of positive real numbers satisfying $\lambda_{n}>M_{n}$ for every $n \in \mathbb{N}$. Then $\overline{\operatorname{conv}}\left(E_{f,\left(\lambda_{n}\right)}\right)=X$.

Proof. By Lemma 3.1, it is sufficient to prove that for any $T \in X^{*} \backslash\{0\}$ and any $r \in \mathbb{R}$,

$$
(T \leq r) \cap E_{f,\left(\lambda_{n}\right)} \neq \emptyset .
$$

Take any fixed $\bar{x} \in T^{-1}(r)$. Since $T \in X^{*} \backslash\{0\}$, there exists $m \in \mathbb{N}$ such that $T$ is bounded on $U_{m}:=\left\{x \in X:\|x\|_{m} \leq 1\right\}$. Define $\|T\|_{m}=\sup \{|T(x)|$ : $\left.x \in U_{m}\right\}$. Then $0<\|T\|_{m}<\infty$. By assumption, $-\infty<\inf f(X)$. Thus for any $x \in X$ and any $y \in X$ with $\|y\|_{m}=1$, we have

$$
\liminf _{t \rightarrow \infty} \frac{f(x+t y)}{t}=\liminf _{t \rightarrow \infty} \frac{f(x+t y)-\inf f(X)}{t} \geq 0
$$

and so $0 \leq M_{n}<\infty$. Since $0 \leq M_{n} / \lambda_{n}<1$, there exists $\bar{y} \in X$ with $\|\bar{y}\|_{m}=1$ such that

$$
\frac{M_{m}}{\lambda_{m}}\|T\|_{m}<T(\bar{y})
$$

that is,

$$
\frac{1}{\lambda_{m}}\left(\sup _{x \in X} \sup _{\substack{y \in X \\\|y\|_{m}=1}} \liminf _{t \rightarrow \infty} \frac{f(x+t y)}{t}\right)\|T\|_{m}<T(\bar{y}) .
$$

In particular,

$$
\frac{1}{\lambda_{m}}\left(\liminf _{t \rightarrow \infty} \frac{f(\bar{x}-t \bar{y})}{t}\right)\|T\|_{m}<T(\bar{y}),
$$

or equivalently,

$$
\frac{1}{\lambda_{m}}\left(\liminf _{t \rightarrow \infty} \frac{f(\bar{x}-t \bar{y})-\inf f(X)}{t}\right)\|T\|_{m}<T(\bar{y}) .
$$


Hence there exists $\bar{t}>0$ such that

$$
\frac{1}{\lambda_{m}} \cdot \frac{f(\bar{x}-\bar{t} \bar{y})-\inf f(X)}{\bar{t}} \cdot\|T\|_{m}<T(\bar{y}) .
$$

Put $\varphi_{m}(x)=(f(x)-\inf f(X)) / \lambda_{m}$. Then (8) becomes

$$
\frac{\varphi_{m}(\bar{x}-\bar{t} \bar{y})}{\bar{t}}<\frac{T(\bar{y})}{\|T\|_{m}} \text {. }
$$

By (9),

$$
T(\bar{y})>\frac{\varphi_{m}(\bar{x}-\bar{t} \bar{y})}{\bar{t}} \cdot\|T\|_{m} \geq 0,
$$

hence $T(\bar{x}-\bar{t} \bar{y})=T(\bar{x})-\bar{t} T(\bar{y})<T(\bar{x})=r$, which means that

$$
\bar{x}-\bar{t} \bar{y} \in(T<r) \text {. }
$$

Again using (9), we obtain

$$
\varphi_{m}(\bar{x}-\bar{t} \bar{y})<\frac{\bar{t} T(\bar{y})}{\|T\|_{m}}=\frac{r-T(\bar{x}-\bar{t} \bar{y})}{\|T\|_{m}} .
$$

Hence for any $x \in T^{-1}(r)$, we have

$$
\varphi_{m}(\bar{x}-\bar{t} \bar{y})<\frac{T(x-(\bar{x}-\bar{t} \bar{y}))}{\|T\|_{m}} \leq\|x-(\bar{x}-\bar{t} \bar{y})\|_{m} .
$$

Put $x_{0}:=\bar{x}-\bar{t} \bar{y}$. Then (10) becomes

$$
x_{0} \in(T<r)
$$

and (11) becomes

$$
\varphi_{m}\left(x_{0}\right) \leq\left\|x-x_{0}\right\|_{m}, \quad \forall x \in T^{-1}(r) .
$$

Assume that there exists a point $y \in X$ such that $\left\|y-x_{0}\right\|_{m} \leq \varphi_{m}\left(x_{0}\right)$ and $y \notin(T \leq r)$. Then $T(y)>r$ and by (12), $T\left(x_{0}\right)<r$. Thus there exists $\theta \in(0,1)$ such that $T\left(\theta x_{0}+(1-\theta) y\right)=r$. Applying (13) to $x=$ $\theta x_{0}+(1-\theta) y \in T^{-1}(r)$, we get

$$
\left\|x_{0}-y\right\|_{m} \leq \varphi_{m}\left(x_{0}\right) \leq\left\|\theta x_{0}+(1-\theta) y-x_{0}\right\|_{m}=(1-\theta)\left\|y-x_{0}\right\|_{m} .
$$

We assert that $\left\|y-x_{0}\right\|_{m}>0$. If not, we have $x_{0}-y \in U_{m} / l$ for all $l>0$, which yields $T\left(x_{0}-y\right)=0$ and $T\left(x_{0}\right)=T(y)$. This contradicts $T\left(x_{0}\right)<r<T(y)$. Since $\left\|y-x_{0}\right\|_{m}>0$, by (14) we have

$$
\left\|x_{0}-y\right\|_{m} \leq(1-\theta)\left\|y-x_{0}\right\|_{m}<\left\|x_{0}-y\right\|_{m},
$$

a contradiction. Thus we have shown that

$$
B_{m}\left(x_{0}, \varphi_{m}\left(x_{0}\right)\right) \subset(T \leq r) .
$$

Here $B_{m}\left(x_{0}, \varphi_{m}\left(x_{0}\right)\right)$ denotes the set $\left\{x \in X:\left\|x-x_{0}\right\|_{m} \leq \varphi_{m}\left(x_{0}\right)\right\}$. Next we consider the following two cases. 
Case (i): $\varphi_{m}\left(x_{0}\right)=0$. Then $\left(f\left(x_{0}\right)-\inf f(X)\right) / \lambda_{m}=0$, which implies that $f\left(x_{0}\right)=\inf f(X)$. Thus for any $x \in X, x \neq x_{0}$,

$$
f(x)>f\left(x_{0}\right)-\sup _{n} \lambda_{n}\left\|x-x_{0}\right\|_{n} .
$$

Obviously $x_{0} \in E_{f,\left(\lambda_{n}\right)}$ and $x_{0} \in(T<r)$, hence $E_{f,\left(\lambda_{n}\right)} \cap(T \leq r) \neq \emptyset$.

Case (ii): $\varphi_{m}\left(x_{0}\right)>0$. Then $\left(f\left(x_{0}\right)-\inf f(X)\right) / \lambda_{m}>0$. For this $m$, by Corollary 2.1 there exists $z \in X$ such that

$$
\lambda_{m}\left\|z-x_{0}\right\|_{m} \leq f\left(x_{0}\right)-f(z), \quad \sup _{n} \lambda_{n}\|x-z\|_{n}+f(x)>f(z), \quad \forall x \neq z .
$$

This implies that

$$
\left\|z-x_{0}\right\|_{m} \leq \frac{f\left(x_{0}\right)-f(z)}{\lambda_{m}} \leq \frac{f\left(x_{0}\right)-\inf f(X)}{\lambda_{m}}=\varphi_{m}\left(x_{0}\right)
$$

and $z \in E_{f,\left(\lambda_{n}\right)}$. Thus $z \in B_{m}\left(x_{0}, \varphi_{m}\left(x_{0}\right)\right) \cap E_{f,\left(\lambda_{n}\right)}$. By (15), we conclude that

$$
z \in(T \leq r) \cap E_{f,\left(\lambda_{n}\right)} \quad \text { and } \quad(T \leq r) \cap E_{f,\left(\lambda_{n}\right)} \neq \emptyset .
$$

Therefore, in any case we always have $E_{f,\left(\lambda_{n}\right)} \cap(T \leq r) \neq \emptyset$. By Lemma 3.1 we have

$$
\overline{\operatorname{conv}}\left(E_{f,\left(\lambda_{n}\right)}\right)=X
$$

Corollary 3.1. Let $(X, \mathcal{T})$ be a Fréchet space with topology generated by an increasing sequence \|\|$_{1} \leq\|\|_{2} \leq \cdots$ of seminorms, and $f:(X, \mathcal{T})$ $\rightarrow \mathbb{R}$ be a lower semicontinuous function, bounded from below, which satisfies

$$
\limsup _{\|x\|_{n} \rightarrow \infty} \frac{f(x)}{\|x\|_{n}}<\infty, \quad \forall n \in \mathbb{N} .
$$

Let $\left(\lambda_{n}\right)_{n \in \mathbb{N}}$ be a sequence of positive real numbers such that

$$
\lambda_{n}>\limsup _{\|x\|_{n} \rightarrow \infty} \frac{f(x)}{\|x\|_{n}}, \quad \forall n \in \mathbb{N} .
$$

Then $\overline{\operatorname{conv}}\left(E_{f,\left(\lambda_{n}\right)}\right)=X$.

Proof. For each $x \in X$ and each $y \in X$ with $\|y\|_{n}=1$, we have

$$
\begin{aligned}
\liminf _{t \rightarrow \infty} \frac{f(x+t y)}{t} & =\liminf _{t \rightarrow \infty} \frac{f(x+t y)-\inf f(X)}{t} \\
& =\liminf _{t \rightarrow \infty} \frac{f(x+t y)-\inf f(X)}{t+\|x\|_{n}} \cdot \frac{t+\|x\|_{n}}{t} \\
& =\liminf _{t \rightarrow \infty} \frac{f(x+t y)-\inf f(X)}{t+\|x\|_{n}}
\end{aligned}
$$




$$
\begin{aligned}
& \leq \liminf _{t \rightarrow \infty} \frac{f(x+t y)-\inf f(X)}{\|x+t y\|_{n}} \\
& \leq \limsup _{\|z\|_{n} \rightarrow \infty} \frac{f(z)-\inf f(X)}{\|z\|_{n}}=\limsup _{\|z\|_{n} \rightarrow \infty} \frac{f(z)}{\|z\|_{n}}<\infty .
\end{aligned}
$$

By Theorem 3.1, we see that $\overline{\operatorname{conv}}\left(E_{f,\left(\lambda_{n}\right)}\right)=X$.

When $(X, \mathcal{T})$ in Theorem 3.1 is a Banach space, we immediately obtain the following consequence.

Corollary 3.2. Let $(X,\|\|)$ be a Banach space and $f:(X,\|\|) \rightarrow \mathbb{R}$ be a lower semicontinuous function, bounded from below, which satisfies

If

$$
\sup _{x \in X} \sup _{\substack{y \in X \\\|y\|=1}} \liminf _{t \rightarrow \infty} \frac{f(x+t y)}{t}<\infty .
$$

then $\overline{\operatorname{conv}}\left(E_{f, \lambda}\right)=X$.

$$
\lambda>\sup _{x \in X} \sup _{\substack{y \in X \\\|y\|=1}} \liminf _{t \rightarrow \infty} \frac{f(x+t y)}{t},
$$

The following example shows that the condition

$$
\sup _{x \in X} \sup _{\substack{y \in X \\\|y\|=1}} \liminf _{t \rightarrow \infty} \frac{f(x+t y)}{t}<\infty
$$

in Corollary 3.2 is strictly weaker than the condition $\lim \sup _{\|x\| \rightarrow \infty} f(x) /\|x\|$ $<\infty$ in Theorem 1.2. Hence Corollary 3.2 is a proper improvement of Theorem 1.2 .

ExAmple 3.1. Let $f: X=\mathbb{R}^{2} \rightarrow \mathbb{R}$ be defined as follows:

$$
f(x, y)= \begin{cases}\frac{|y|^{3}}{\left(y-x^{2}\right)^{2}+x^{2}} & \text { if }(x, y) \neq(0,0), \\ 0 & \text { if }(x, y)=(0,0) .\end{cases}
$$

Let $x=r \cos \theta, y=r \sin \theta(r>0,0 \leq \theta<2 \pi)$. Then for $(x, y) \neq(0,0)$, as $r=\sqrt{x^{2}+y^{2}} \rightarrow 0$,

$$
\begin{aligned}
f(x, y) & =\frac{|r \sin \theta|^{3}}{\left(r \sin \theta-r^{2} \cos ^{2} \theta\right)^{2}+r^{2} \cos ^{2} \theta} \\
& =\frac{r\left|\sin ^{3} \theta\right|}{1-2 r \sin \theta \cos ^{2} \theta+r^{2} \cos ^{4} \theta} \rightarrow 0 .
\end{aligned}
$$

Thus $\lim _{(x, y) \rightarrow(0,0)} f(x, y)=0=f(0,0)$. Hence $f$ is continuous on $\mathbb{R}^{2}$ and clearly $f$ is bounded from below. Let $\mathbb{R}^{2}$ be endowed with the Euclidean norm, i.e., $\|(x, y)\|=\sqrt{x^{2}+y^{2}}$ for $(x, y) \in \mathbb{R}^{2}$. Take any point $\left(x_{0}, y_{0}\right) \in$ 
$X=\mathbb{R}^{2}$ and any point $(\cos \theta, \sin \theta)$ in the unit sphere. We consider

$$
\liminf _{t \rightarrow \infty} \frac{f\left(\left(x_{0}, y_{0}\right)+t(\cos \theta, \sin \theta)\right)}{t},
$$

according to the following two cases.

CASE 1: $\cos \theta \neq 0$. Then for any $t>0$,

$$
\begin{aligned}
& \frac{f\left(\left(x_{0}, y_{0}\right)+t(\cos \theta, \sin \theta)\right)}{t}=\frac{f\left(x_{0}+t \cos \theta, y_{0}+t \sin \theta\right)}{t} \\
& =\frac{1}{t} \cdot \frac{\left|y_{0}+t \sin \theta\right|^{3}}{\left[y_{0}+t \sin \theta-\left(x_{0}+t \cos \theta\right)^{2}\right]^{2}+\left(x_{0}+t \cos \theta\right)^{2}} \\
& =\frac{1}{t^{2}} \cdot \frac{\left|\frac{1}{t} y_{0}+\sin \theta\right|^{3}}{\left[\frac{1}{t^{2}} y_{0}+\frac{1}{t} \sin \theta-\left(\frac{1}{t} x_{0}+\cos \theta\right)^{2}\right]^{2}+\left(\frac{1}{t^{2}} x_{0}+\frac{1}{t} \cos \theta\right)^{2}} .
\end{aligned}
$$

From this, we easily see that

$$
\lim _{t \rightarrow \infty} \frac{f\left(\left(x_{0}, y_{0}\right)+t(\cos \theta, \sin \theta)\right)}{t}=0 \cdot \frac{\left|\sin ^{3} \theta\right|}{\cos ^{4} \theta}=0 .
$$

Case 2: $\cos \theta=0$. Then $\sin \theta= \pm 1$. Thus

$$
\begin{aligned}
\frac{f\left(\left(x_{0}, y_{0}\right)+t(\cos \theta, \sin \theta)\right)}{t} & =\frac{f\left(x_{0}, y_{0} \pm t\right)}{t} \\
& =\frac{1}{t} \cdot \frac{\left|y_{0} \pm t\right|^{3}}{\left(y_{0} \pm t-x_{0}^{2}\right)^{2}+x_{0}^{2}} \\
& =\frac{\left|\frac{1}{t} y_{0} \pm 1\right|^{3}}{\left(\frac{1}{t} y_{0} \pm 1-\frac{1}{t} x_{0}^{2}\right)^{2}+\frac{1}{t^{2}} x_{0}^{2}} \rightarrow 1
\end{aligned}
$$

as $t \rightarrow \infty$. Therefore $f$ is a continuous function, bounded from below, which satisfies

$$
\sup _{u \in X} \sup _{\substack{v \in X \\\|v\|=1}} \liminf _{t \rightarrow \infty} \frac{f(u+t v)}{t} \leq 1<\infty .
$$

However, we shall see that

$$
\limsup _{\|(x, y)\| \rightarrow \infty} \frac{f(x, y)}{\|(x, y)\|}=\infty .
$$

In fact, let $(x, y)$ go to infinity along the curve $y=x^{2}$; then

$$
\lim _{\substack{y=x^{2} \\ x \rightarrow \infty}} \frac{f(x, y)}{\|(x, y)\|}=\lim _{x \rightarrow \infty} \frac{x^{4}}{|x| \sqrt{1+x^{2}}}=\infty .
$$

4. The existence and density of Caristi fixed points. Using Ekeland's variational principle, some authors considered various extensions of Caristi's fixed point theorem [2]; for example see Cammaroto and Chinni [1], Ekeland [5], Fang [6], Mizoguchi [8] and Penot [10]. Here we derive from 
Theorems 2.1 and 3.1 the corresponding results concerning the existence and density of Caristi fixed points.

THEOREM 4.1. Let $(X, \mathcal{T})$ be a Fréchet space with topology generated by an increasing sequence \|\|$_{1} \leq\|\|_{2} \leq \cdots$ of seminorms and $\left(\varphi_{n}\right)_{n \in \mathbb{N}}$ be a sequence of functions in $\Theta$ (concerning $\Theta$, see $\S 2)$. Assume that $f:(X, \mathcal{T}) \rightarrow$ $\mathbb{R} \cup\{\infty\}$ is a lower semicontinuous proper function, bounded from below, and that $T: X \rightarrow X$ is a multivalued mapping such that for every $n$,

$$
\varphi_{n}\left(\|x-y\|_{n}\right)+f(y) \leq f(x), \quad \forall x \in X, \forall y \in T x
$$

Then there exists a $z \in X$ such that $T z=\{z\}$.

Proof. By Theorem 2.1, there exists $z \in \operatorname{dom}(f)$ such that

$$
\sup _{n} \varphi_{n}\left(\|x-z\|_{n}\right)+f(x)>f(z), \quad \forall x \neq z .
$$

If $T z \neq\{z\}$, then there exists $y \in T z$ such that $y \neq z$. By (16), we have

$$
\sup _{n} \varphi_{n}\left(\|y-z\|_{n}\right)+f(y)>f(z) \text {. }
$$

From this, there exists $m \in \mathbb{N}$ such that

$$
\varphi_{m}\left(\|y-z\|_{m}\right)+f(y)>f(z) \text {. }
$$

On the other hand, by the assumption on the multivalued mapping $T$, we have

$$
\varphi_{n}\left(\|y-z\|_{n}\right)+f(y) \leq f(z), \quad \forall n \in \mathbb{N} .
$$

This contradicts (17). Thus we have shown that $T z=\{z\}$.

In particular, the result of Theorem 4.1 remains true if the function sequence $\left(\varphi_{n}\right)_{n \in \mathbb{N}}$ is replaced by a positive real number sequence $\left(\lambda_{n}\right)_{n \in \mathbb{N}}$ and the assumption on the multivalued mapping $T$ is replaced by the assumption that for every $n$,

$$
\lambda_{n}\|x-y\|_{n}+f(y) \leq f(x), \quad \forall x \in X, \forall y \in T x
$$

Similarly, using Theorem 3.1 we can easily obtain the following density result for Caristi fixed points.

THEOREM 4.2. Let $(X, \mathcal{T})$ be a Fréchet space with topology generated by an increasing sequence \|\|$_{1} \leq\|\|_{2} \leq \cdots$ of seminorms. Let $f:(X, \mathcal{T}) \rightarrow \mathbb{R}$ be a lower semicontinuous function, bounded from below and satisfying

$$
M_{n}:=\sup _{x \in X} \sup _{\substack{y \in X \\\|y\|_{n}=1}} \liminf _{t \rightarrow \infty} \frac{f(x+t y)}{t}<\infty, \quad \forall n \in \mathbb{N},
$$

and let $\left(\lambda_{n}\right)_{n \in \mathbb{N}}$ be a sequence of positive real numbers such that $\lambda_{n}>M_{n}$ for all $n \in \mathbb{N}$. If $T: X \rightarrow X$ is a multivalued mapping such that for every $n$,

$$
\lambda_{n}\|x-y\|_{n}+f(y) \leq f(x), \quad \forall x \in X, \forall y \in T x
$$

then $\overline{\operatorname{conv}}\{z \in X: T z=\{z\}\}=X$. 
By Corollary 3.2, we have the following.

Corollary 4.1. Let $(X,\|\|)$ be a Banach space and $f:(X,\|\|) \rightarrow \mathbb{R}$ be a lower semicontinuous function, bounded from below, which satisfies

$$
M:=\sup _{x \in X} \sup _{\substack{y \in X \\\|y\|=1}} \liminf _{t \rightarrow \infty} \frac{f(x+t y)}{t}<\infty .
$$

If a positive real number $\lambda>M$ and a multivalued mapping $T: X \rightarrow X$ satisfy

$$
\lambda\|x-y\|+f(y) \leq f(x), \quad \forall x \in X, \forall y \in T x,
$$

then $\overline{\operatorname{conv}}\{z \in X: T z=\{z\}\}=X$.

The author would like to thank the referee for his valuable comments and suggestions.

\section{References}

[1] F. Cammaroto and A. Chinni, A complement to Ekeland's variational principle in Banach spaces, Bull. Polish Acad. Sci. Math. 44 (1996), 29-33.

[2] J. Caristi, Fixed point theorems for mappings satisfying inwardness conditions, Trans. Amer. Math. Soc. 215 (1976), 241-251.

[3] S. Dolecki and S. Kurcyusz, On $\Phi$-convexity in extremal problems, SIAM J. Control Optim. 16 (1978), 277-300.

[4] I. Ekeland, On the variational principle, J. Math. Anal. Appl. 47 (1974), 324-353.

[5] -, Nonconvex minimization problems, Bull. Amer. Math. Soc. 1 (1979), 443-474.

[6] J. X. Fang, The variational principle and fixed point theorems in certain topological spaces, J. Math. Anal. Appl. 202 (1996), 398-412.

[7] G. Köthe, Topological Vector Spaces I, Springer, Berlin, 1983.

[8] N. Mizoguchi, A generalization of Brøndsted's results and its applications, Proc. Amer. Math. Soc. 108 (1990), 707-714.

[9] D. Pallaschke and S. Rolewicz, Foundations of Mathematical Optimization, Math. Appl. 388, Kluwer, Dordrecht, 1997.

[10] J. P. Penot, The drop theorem, the petal theorem and Ekeland's variational principle, Nonlinear Anal. 10 (1986), 813-822.

[11] R. R. Phelps, Convex Functions, Monotone Operators and Differentiability, Springer, Berlin, 1989.

[12] S. Rolewicz, On $\Phi$-differentiability of functions over metric spaces, Topol. Methods Nonlinear Anal. 5 (1995), 229-236.

Department of Mathematics

Suzhou University

Suzhou, Jiangsu 215006

People's Republic of China

E-mail: qjhsd@sina.com.cn 